\title{
Comparing Smooth Arm Movements with the Two-Thirds Power Law and the Related Segmented-Control Hypothesis
}

\author{
Magnus J. E. Richardson ${ }^{1,2}$ and Tamar Flash ${ }^{1}$ \\ ${ }^{1}$ Department of Applied Mathematics, Weizmann Institute of Science, Rehovot 76200, Israel, and 2Laboratoire de \\ Physique Statistique, Ecole Normale Supérieure, 75231, Paris Cedex 05, France
}

\begin{abstract}
The movements of the human arm have been extensively studied for a variety of goal-directed experimental tasks. Analyses of the trajectory and velocity of the arm have led to many hypotheses for the planning strategies that the CNS might use. One family of control hypotheses, including minimum jerk, snap and their generalizations to higher orders, comprises those that favor smooth movements through the optimization of an integral cost function. The predictions of each order of this family are examined for two standard experimental tasks: point-topoint movements and the periodic tracing of figural forms, and compared both with experiment and the two-thirds power law. The aim of the analyses is to generalize previous numerical observations as well as to examine movement segmentation. It is first shown that contrary to recent statements in the literature, the only members of this family of control theories that match
\end{abstract}

reaching movement experiments well are minimum jerk and snap. Then, for the case of periodic drawing, both the ellipse and cloverleaf are examined and the experimentally observed power law is derived from a first-principles approach. The results for the ellipse are particularly general, representing a unification of the two-thirds power law and smoothness hypotheses for ellipses of all reasonable eccentricities. For complex shapes it is shown that velocity profiles derived from the cost-function approach exhibit the same experimental features that were interpreted as segmented control by the CNS. Because the cost function contains no explicit segmented control, this result casts doubt on such an interpretation of the experimental data.

Key words: motor control; two-thirds power law; arm; planning strategies; cost function; minimum jerk; segmentation
Movements of the human body are restricted both by mechanics and computational resources. However, the repertoire of movements that is actually observed comprises a small part of those that are possible, because the CNS uses planning strategies that impose further restrictions. The determination of these strategies is nontrivial because they are not measurable directly. The approach has been therefore to observe the behavior under various conditions and to construct mathematical theories that describe the output of the motor system. The past few decades of experimentation have lead to a number of (apparently) different hypotheses for planning strategies; for example, it has been proposed that the CNS plans in the coordinate system of the joint angles (Uno et al., 1989; Nakano et al., 1999) or in the coordinates of the hand's position (Morasso, 1981; Flash and Hogan, 1985) or that the CNS plans movements that are robust against the inherent noise in the motor system (Harris, 1998; Harris and Wolpert, 1998).

A successful description of an experiment by a theory is usually taken as evidence that the CNS does indeed operate in such a manner. But what if two different theories have the same predictions? Obviously in this case no conclusions can be firmly drawn until further experiments are performed, outside the region of agreement. However, hypotheses can also be compared mathe-

Received Nov. 27, 2001; revised April 23, 2002; accepted May 17, 2002.

This work was supported in part by the Minerva Foundation (Germany), by a grant from the Israeli Ministry of Science, Culture, and Sport, and by the Moross Laboratory. M.J.E.R. acknowledges the support from a Feinberg research fellowship.

Correspondence should be addressed to Magnus J. E. Richardson, Laboratoire de Physique Statistique, Ecole Normale Superieure, 24 Rue Lhomond 75231, Paris Cedex 05, France. E-mail: Magnus.Richardson@lps.ens.fr.

Copyright (C) 2002 Society for Neuroscience $0270-6474 / 02 / 228201-11 \$ 15.00 / 0$ matically to reach an understanding of their interrelation. In this paper such a comparative study is undertaken with respect to two simple, but widely applicable theories: the optimization of smoothness-based cost functions and the two-thirds power law between curvature and velocity. Predictions of these control hypotheses will be examined for two standard experimental paradigms: point-to-point reaching movements and the periodic tracing of simple figures. For the case of point-to-point movements it is shown that only a few members of the family of smoothnessbased cost functions can match experiments well. For the case of the periodic drawing of figural forms, a number of novel results will be derived. A mathematical form for the exponent relating hand velocity to curvature of the two-thirds power law will be obtained from the cost functions for an ellipse and shown to be indistinguishable from the experimental value of $\sim 0.33$ (the name "two-thirds" power law comes from the original formulation in terms of curvature and angular velocity). This result holds for all practical eccentricities, generalizing and formalizing previous numerical evidence obtained for specific examples (Wann et al. 1988; Harris and Wolpert, 1998). As has been pointed out previously, the ellipse is a special case with respect to the twothirds power law because it implies coupled harmonic motion. For this reason, the same analysis was performed for the cloverleaf, and again, an exponent was found that was in good agreement with experiment. Finally, the case of movement segmentation in complex shapes is examined. The velocity-curvature relationship is derived from the smoothness-based cost function for a figureof-eight. It is shown that the same features of segmentation seen in experiment are seen in the mathematically derived velocity profile. Because the cost function contains no segmented control, this suggests that this apparent segmentation is an epiphenome- 
num of smooth movements, in agreement with the experimental work of Sternad and Schaal (1999).

\section{MATERIALS AND METHODS}

In this section, the two motor-control hypotheses to be studied will be defined mathematically, and the notion of segmentation with respect to the two-thirds power law will be reviewed.

The mean squared derivative cost functions. Each member of this family of motion-planning hypotheses is a generalized version of the minimumjerk approach used initially to model velocity profiles generated by elbow movements (Hogan, 1984) and later extended to trajectory prediction for reaching movements between visual targets in the horizontal plane and to curved and obstacle-avoidance movements (Flash and Hogan, 1985). Since then, the minimum-jerk hypothesis has been applied to a great many other motor tasks, including the drawing of complicated figural patterns (Viviani and Flash, 1995; Todorov and Jordan, 1998) for which a numerical approach was used to obtain velocity profiles in excellent agreement with experiment. The predictions of these mean squared derivative (MSD) hypotheses are derived through the minimization of the time integral of the squared $n$ th-derivative of the coordinates of the hand $(x(t), y(t))$ :

$$
C_{n}=\int_{0}^{T} d t\left(\left(\frac{d^{n} x}{d t^{n}}\right)^{2}+\left(\frac{d^{n} y}{d t^{n}}\right)^{2}\right)
$$

where $T$ is the duration, or period of the movement. Each order $n$, which can take any integer value between unity and infinity, corresponds to a different member of the family and is an independent hypothesis for how the CNS might plan movement. For $n=2, n=3$, and $n=4$ the corresponding hypotheses have been named minimum acceleration, jerk, and snap, respectively. The case $n=1$ of minimum velocity is not compatible with the observed velocity and acceleration data and will not be considered here.

For completeness it should be stated that the trajectories derived miss some of the fine detail seen in point-to-point movements. The paths measured are actually slightly, but systematically, curved depending on their position with respect to the body. This observation has led to further hypotheses (Flash, 1987; Uno et al., 1989; Harris and Wolpert, 1998) that deal with the problem of motor execution and include explicit details of the mechanical properties of the arm. Nevertheless, the simpler cost function of minimum jerk gives a satisfactory prediction of the observed movements and is more than sufficiently accurate to provide the basis for the analyses below.

Given that minimum jerk is just one member of the MSD cost function family (with $n=3$ ), it could be asked if the theoretical velocity profiles derived for other orders give an equally good prediction of experiment. Recently it was stated in the literature (Harris, 1998) that this was indeed the case. If this were true it would of course imply a fundamental problem with MSD-generated velocity profiles: if they all fit the data equally well there are no criteria for choosing one hypothesis over another. In the first part of Results it will be shown that in fact this is not the case. By calculating the predicted velocity profiles for each order, it will be shown that only minimum jerk (and to a lesser extent minimum snap) is compatible with the experimental data.

The two-thirds power law. In the extensive analysis of handwriting and drawing motion, where the trajectories of the hand are curved, it was seen that the velocity is not constant but varies strongly with the instantaneous curvature. It was found (Viviani and Terzuolo, 1982; Lacquaniti et al., 1983) that this phenomena could be expressed in terms of a power law between velocity $v$ and curvature $\kappa$ as follows:

$$
v=g \kappa^{-\beta},
$$

where the accepted value of the exponent $\beta \simeq 0.33$. The factor $g$ is known as the gain factor and is set by the tempo of the movement. The name "two-thirds" power law comes from the original formulation of the law in terms of the angular velocity. The tracing of ellipses of various eccentricities is the most extensively analyzed task used in the examination of the power law. However, many results exist for more complex shapes, and although the power is not indistinguishable from 0.33 in every case (Wann et al. 1988; Viviani and Schneider, 1991; Viviani and Flash, 1995) the power-law form still holds with an exponent close to this value.

An intriguing aspect of the power law is seen in the tracing of extended shapes and also in free scribbling. In such cases a single value of the gain factor $g$ is not sufficient to fit the data. However, a power law between velocity and curvature can still be shown to exist if a piecewise constant gain factor is used. A second and separate issue is that the regions of zero curvature in such shapes are incompatible with the power law in its usual form, and so an altered form involving an effective radius of curvature $R^{*}$ (the standard radius of curvature is $R=1 / \kappa$ ) was also introduced to avoid divergences (Viviani and Stucchi, 1992). Thus the velocity $v$, in this case, takes the form:

$$
v=g_{j}\left(R^{*}\right)^{\beta}=g_{j}\left(\frac{R}{1+\alpha R}\right)^{\beta} \text { for each segment } j \text { of the shape, }
$$

with $\alpha=0.05$. The discontinuous changes throughout the movement of the gain factor are compelling evidence for segmentation of movements in the CNS (Viviani and Cenzato, 1985) and are seen clearly in log-log plots of velocity versus curvature. Recently, this view of segmentation was challenged in the paper of Sternad and Schall (1999). Subjects were asked to trace increasingly large ellipses with the point of their finger. It was found that the larger the ellipse traced, the clearer the evidence of segmentation in the velocity of the finger. However, at the same time it was shown that the motion at the joints was purely oscillatory. Based on these observations, it was concluded that the apparent segmentation of the hand trajectories was a result of the nonlinearities in the forward kinematics of the human arm (which become significant for large movements). However, it is also true that segmentation is seen in the tracing of small, but complex shapes, which would be in the linear regime of the kinematic transformations. The aim here will be to examine if a smoothness maximization of the end-point trajectory around such a complex shape also yields apparent segmentation [it should be stressed at this point that unlike in Viviani and Flash (1995), no experimental data is used in obtaining the prediction: the approach is from first principles].

\section{RESULTS}

In this section, explicit mathematical forms for the order- $n$ predicted velocity profiles will be given for the two contrasting experimental tasks of point-to-point and periodic drawing movements.

\section{Reaching movements in the plane}

The aim of this section is to analyze the forms of the predicted velocity profiles for each order $n$ of the derivative of the coordinates of the hand. The mathematical form for the $n$ th-order profile will be obtained, and the limit $n \rightarrow \infty$ will be examined. It will be shown that the profiles diverge in this limit, and therefore not all MSD profiles can fit the data equally well.

The position vector of the hand is written as $r=(x, y)$. Using the Euler-Lagrange formalism for the functional minimization of the cost function given in Equation 1 yields the following differential equation for the trajectory $r$ as a function of time $t$ :

$$
\frac{d^{2 n} r}{d t^{2 n}}=0
$$

This implies that $r$ is an order $(2 n-1)$ th polynomial in time and therefore that the velocity is an order $(2 n-2)$ th polynomial in time. The constants in this polynomial can be found by applying the boundary conditions. The first set of boundary conditions are those that enforce the maximal allowable stationarity at the start and at the end of the movement. For an $n$th order MSD velocity profile this requires setting the first $(n-1)$ th time derivatives of $r$ to zero at $t=0$ and $t=T$. As an example, minimum jerk with $n=3$ has boundary conditions of zero velocity and acceleration at the start and end of the reach. It should be noted that these boundary conditions are the only choice that does not require input of experimental data (e.g. the velocity) at the beginning and end of the movement. The differential Equation 4 for the trajectory can be integrated using the boundary conditions, allowing all 
but one of the $2 n-1$ constants in the velocity profile $v_{n}$ to be found:

$$
v_{n}=p_{n}(4 \tau(1-\tau))^{n-1},
$$

where the only unknown is the peak velocity $p_{n}$ (occurring at $\tau=$ $1 / 2$ ) which is different for each order $n$ of the cost function. The notation $\tau=t / T$ (where $T$ is the movement duration) has also been introduced to simplify the appearance of the equation. The final quantity needed, $p_{n}$, can be found by integrating the velocity over time and setting the result equal to the total amplitude of the movement $L$. The resulting integral can be found in a standard book of tables and gives the following form for the peak velocity, and its limit when $n$ is large:

$$
\begin{aligned}
& p_{n}=\left(\frac{L}{T}\right) \frac{1}{4^{n-1}} \frac{(2 n-1) !}{(n-1) !^{2}} \\
& p_{n} \simeq 2\left(\frac{L}{T}\right) \sqrt{\frac{n}{\pi}} \text { for } n \rightarrow \infty .
\end{aligned}
$$

The $n \rightarrow \infty$ limit was obtained using Stirling's formula for factorials of large numbers. This result should be interpreted as the average velocity $L / T$ multiplied by an order- $n$-dependent factor. The ratio between the peak and average velocities implied by this result is a simple (but sufficient for the purposes of discounting the higher-order MSD cost functions) measure of the shape of the order- $n$ velocity profile. Experimentally, the mean value of the peak to average velocity ratio has been measured over 30 movements and found to be close to 1.8 (Flash and Hogan, 1985) with an SD of $\sim 10 \%$. This value should be compared with the predicted results from Equation 5:

$$
\begin{array}{cc}
1.5 & \text { minimum acceleration } n=2 \\
1.875 & \text { minimum jerk } n=3 \\
2.186 & \text { minimum snap } n=4
\end{array}
$$

Clearly the predictions of the minimum-jerk cost function fall between the bounds mentioned above, but as can be seen in Figure 1, minimum snap also gives a reasonable fit of the data. From this figure and also Equation 5 it can be seen that the ratio of peak to average velocity increases as a function of $n$. This is a result of the large $n$ form given in Equation 5 above. It is therefore apparent that high-order MSD velocity profiles are incompatible with experiment: because as $n$ tends to infinity the predicted peak velocity goes to infinity, diverging with the squareroot of $n$. The functional form of the velocity profile in this limit can be found using the following simple argument: the distance traveled by the hand is constrained to be $L$ and is equal to the area under the velocity curve. Therefore to compensate for the diverging peak velocity, the width of the bell-shaped velocity profile must become increasingly small for larger $n$ (although still remaining centered at $\tau=1 / 2$ ). In the extreme limit of $n \rightarrow \infty$ this forces the velocity profile to have a vanishingly small width to counterbalance the increasingly high peak velocity. Therefore the velocity curve takes the form of a Dirac delta function centered on time $T / 2$, which is clearly unacceptable experimentally and physically. This result provides the proof that only the low-order MSD velocity profiles could be compatible with experiment.

However, the conclusions reached above need to be reconciled with the results previously reported that stated that the MSD velocity profiles converge to an experimentally more realistic Gaussian function in the large $n$ limit (Harris, 1998). There the velocity profiles found at each order $n$ were rescaled by different

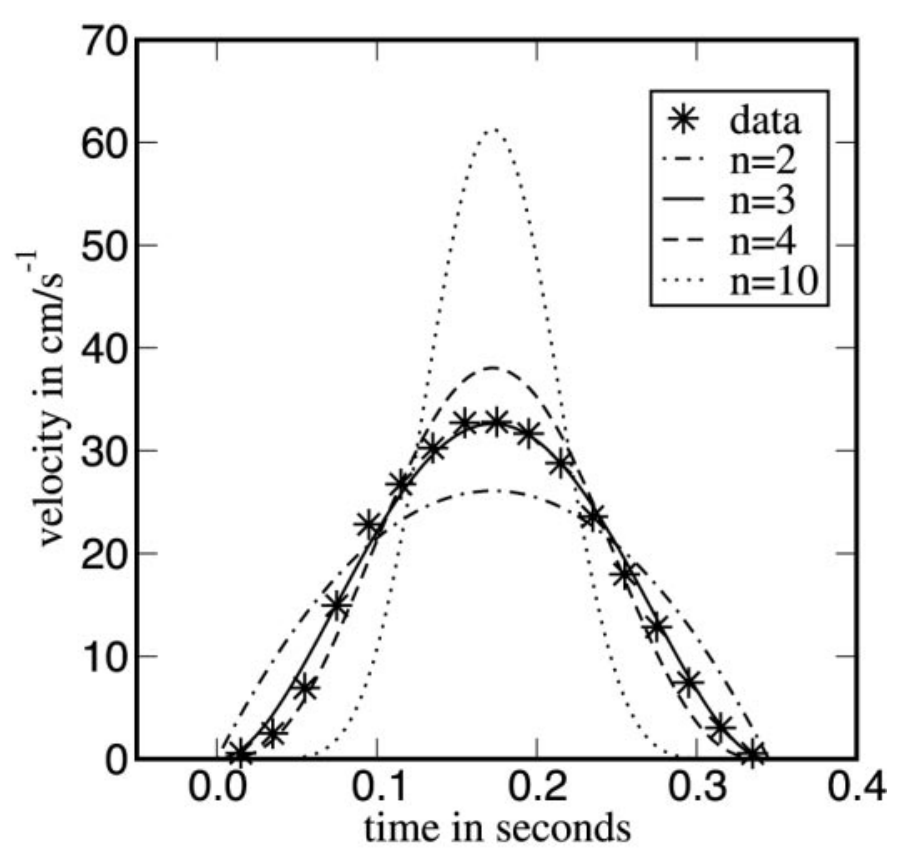

Figure 1. The MSD profiles for $n=2,3,4$, and 10 with the only experimental input being the time scale $T$ (given by the experimental data). The profiles become narrower and taller as $n$ increases. The experimental curve is best fitted by minimum jerk $n=3$ and $\operatorname{snap} n=4$.

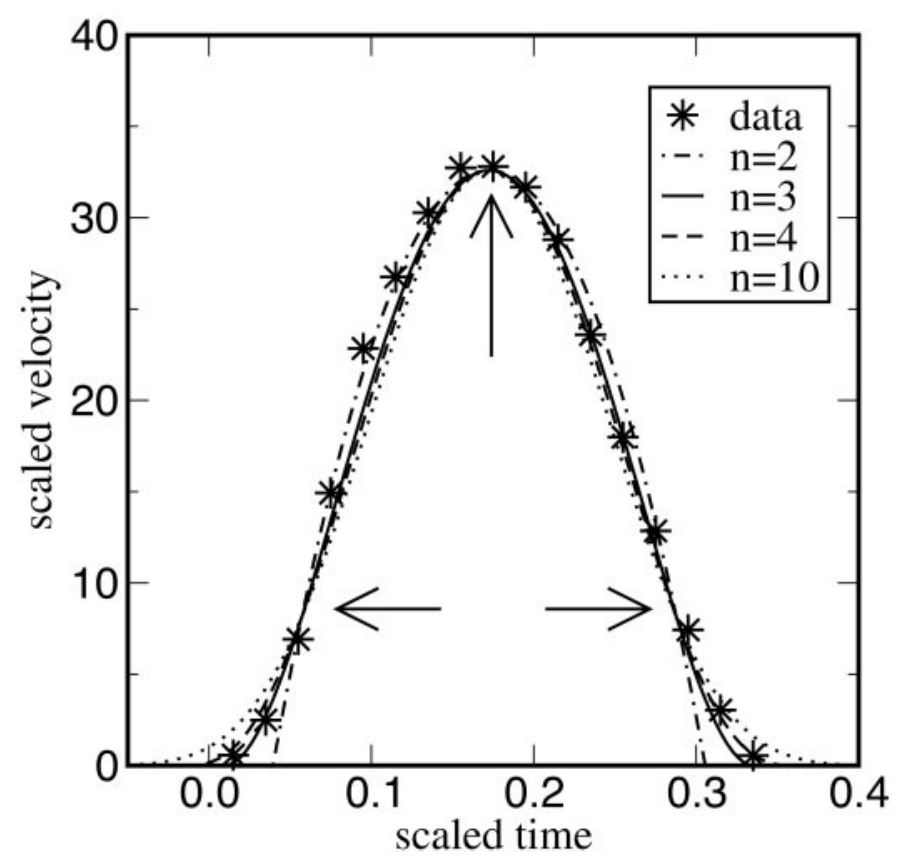

Figure 2. The same profiles as in Figure 1 but rescaled as by Harris (1998). Each profile is rescaled such that they all pass through the points marked by arrows. A cut-off for the start and end of the movements must also be supplied.

(n-dependent) amounts so that their peaks all coincided at the same point and their widths passed through a value of $25 \%$ of the normalized peak velocity at the same times. Such a rescaling of the profiles in Figure 1 has been performed, with the results plotted in Figure 2. In this way, it appears that the curves become similar as $n$ takes higher values, with the $n=\infty$ limit converging to a Gaussian. Nevertheless, because the velocity tails become 
increasingly large, a cut-off must also be introduced to fix the time of the movement to be $T$ (which is given by experiment). Essentially what that approach amounted to was to treat each order- $n$ MSD velocity profile as a curve that can be fitted to experiment by stretching the time axis and compressing the velocity axis. Curve-fitting is a valid and useful approach for examining the shape of the experimental curves (Plamondon et al., 1993). However, it is in spirit a very different approach to the derivation of a curve from a hypothesis as was done here and elsewhere (Flash and Hogan, 1985), with a minimal number of free parameters.

\section{Periodic tracing of figural forms}

In this section the case of continuous drawing of closed shapes is analyzed. The approach will be to substitute into the MSD cost function, written in Equation 1, a specific path (the template of the shape) and then use a minimization procedure to derive the predicted velocity profile. It is important to note that this approach differs from the one taken in an earlier study (Viviani and Flash, 1995) where the experimental values of the velocity of the hand and acceleration at several via-points were used in the minimumjerk prediction. By contrast, in the present study the velocity profiles are derived from a first-principles approach for general classes of shapes, necessitating minimal experimental input (only the path itself and the tempo). In fact the mathematical approach used here is more closely related to the detailed numerical study used in Todorov and Jordan (1998) in which the jerk was minimized along a prescribed path.

In contrast to the previous experimental task, the motion is periodic, and because of this it is natural to describe the motion in the language of Fourier series. The time dependence of the $x$ and $y$ coordinates of the hand can each be expanded in Fourier series, where for convenience the normalized time $\tau=t / T$ is again used (where $T$ is now the period of the motion):

$$
\begin{gathered}
x(\tau)=\frac{a_{0}}{2}+\sum_{k=1}^{\infty}\left[a_{k} \cos (2 \pi k \tau)+b_{k} \sin (2 \pi k \tau)\right] \\
y(\tau)=\frac{p_{0}}{2}+\sum_{k=1}^{\infty}\left[p_{k} \cos (2 \pi k \tau)+q_{k} \sin (2 \pi k \tau)\right] .
\end{gathered}
$$

These forms are quite general and can be used to describe the continuous and repeated tracing of any closed shape. The infinite set of variables $\{a, b, p, q\}$ are fixed both by the path and the velocity profile along the path taken by the hand. When the $x$ and $y$ coordinates are written in this way, the integral (Eq. 1) can be performed to give:

$$
C_{n}=\frac{T}{2} \frac{1}{T^{2 n}} \sum_{k=1}^{\infty}(2 \pi k)^{2 n}\left(a_{k}^{2}+b_{k}^{2}+p_{k}^{2}+q_{k}^{2}\right) .
$$

The Fourier coefficients are of course subject to many auxiliary conditions that ensure that the path taken has the given shape, which still makes minimization very difficult. Nevertheless, this form for the cost function allows for a straightforward and revealing analysis in terms of the frequency components present in the movement. If the first few terms of the series (Eq. 7) are written out explicitly, it becomes clear that the higher-order terms contribute a cost that is amplified by a factor of $k^{2 n}$. This means that, if the cost function is to be minimized, the higher-order Fourier coefficients must be small (as would be expected for smooth movements). In fact, just restricting the cost to be finite means that the square of these coefficients must decay with the order $k$ of the expansion faster than $1 / k^{2 n}$. For low order- $n$ MSD derivative cost functions like acceleration and jerk there is some balance between this amplifying factor and the magnitude of the Fourier coefficients. However, for the higher-order MSD profiles the amplifying factor becomes increasingly significant. This means that in the limit $n \rightarrow \infty$ the corresponding order $n$ MSD trajectory converges to the one that has the smallest allowable values of Fourier coefficients in the range $k>2$. This is the central result of this section: in contrast to the case of point-topoint movements, for periodic movements the limit $n \rightarrow \infty$ predicts a convergence to the maximally smooth velocity profile that follows the given template. This result often allows the minimizing trajectory for the limit $n \rightarrow \infty$ to be found with little mathematical effort. Moreover, as the MSD velocity profiles converge in this case it also provides a good first guess for the lower-order $n$ profiles. This first guess can be used for numerical minimization because it would reduce the time taken to search through the space of possible profiles. The implications of this mathematical analysis are examined for two simple figural forms: the ellipse and cloverleaf.

\section{The ellipse}

The general parametric equations for an ellipse are:

$$
x(\tau)=A \cos (\theta(\tau)) \quad y(\tau)=B \sin (\theta(\tau)),
$$

where for various values of the constants $A$, and $B$ and the function $\theta(\tau)$ the Fourier expansion coefficients in Equations 6 take different values. In particular, the simplest choice of $\theta=2 \pi \tau$ would imply $a_{1}=A, q_{1}=B$, and all other Fourier coefficients would be zero. The Fourier expansion corresponding to this choice has the lowest frequency components in the range $k>2$ (they are all zero), and therefore following the reasoning above, this choice for $\theta$ defines a velocity profile that minimizes the MSD cost function in the limit $n \rightarrow \infty$. As is well known (Wann et al., 1988) this harmonic form:

$$
x(\tau)=a_{1} \cos (2 \pi \tau) \quad y(\tau)=q_{1} \sin (2 \pi \tau)
$$

automatically satisfies the power law between velocity and curvature given in Equation 2 with $\beta=\frac{1}{3}$. Despite the simplicity of the harmonic forms of Equations 9 this is an important result because it unifies mathematically the predictions of the two-thirds power law and the MSD cost function in the limit $n \rightarrow \infty$ for ellipses. This is despite the fact that the two laws were originally formulated independently and have different "philosophical" underpinnings: the two-thirds power law is an empirical law relating instantaneous velocity to the local curvature, whereas the MSD cost function implies the full trajectory is planned as a whole before implementation, by virtue of the integral formulation and was originally designed to model point-to-point movements.

This equivalence between the two laws only holds for the somewhat abstract limit of $n \rightarrow \infty$. It would be more interesting to know what the predictions for the exponent $\beta$ are from MSD hypotheses with orders that are more closely related to quantities with physical meaning (as was shown for point-to-point movements) like jerk or snap. In the remainder of this section, the results of such a calculation are presented with the mathematical details given in Appendix A. The method used is a perturbative approach whereby the calculation is performed for ellipses that are very close to circles, with a small parameter $\epsilon$ measuring deviation of the ellipse from a circle. After performing this 
calculation, the power-law exponent predicted from the order- $n$ MSD cost function can be found to have the following form:

$$
\beta_{n}=\frac{1}{3}-\frac{8}{3\left(9^{n}-1\right)}+\text { corrections of order } \epsilon^{2}
$$

It is clear from the above form that as $n \rightarrow \infty$ the exponent tends to the idealized value of $\frac{1}{3}$ as was argued above. However, this does not mean that the $n \rightarrow \infty$ is the most appropriate order to choose. The numerical values of the exponents predicted by the lower-order $n$ MSD cost functions are given from Equation 10 as:

$$
\begin{gathered}
\beta_{2}=\frac{3}{10}=0.3000 \text { minimum acceleration } \\
\beta_{3}=\frac{30}{91}=0.3297 \text { minimum jerk } \\
\beta_{4}=\frac{273}{820}=0.3329 \text { minimum snap. }
\end{gathered}
$$

The latter two, jerk and snap, are experimentally indistinguishable from 0.33 . In fact all orders $n>2$ predict an exponent that is compatible with this accepted value. It is interesting to note that it was previously thought that only minimum jerk would give predictions similar to the two-thirds power law by virtue of the form of the jerk normal to the velocity tangent vector. As has been shown (Todorov and Jordan, 1998), setting the normal jerk to zero automatically enforces the $\frac{1}{3}$ power-relation between curvature and velocity. However, the result (Eq. 10) shows that the close relation to the power law is present for almost all order- $n$ MSD velocity profiles and is not just a consequence of the special form of the normal jerk for the order $n=3$ MSD cost function. It should be further noted that the theoretical result exhibits other properties seen in experiment. The exponent derived is independent of the size of the ellipse and also the time period of the drawing of the ellipse. More significantly, the predicted exponent is, for practical purposes, independent of the eccentricity of the ellipse by virtue of the weak dependence of $\beta_{n}$ on $\epsilon$ (see Appendix A). Taken together, these features represent a robust reproduction of the experimental results by a first-principles approach.

\section{The cloverleaf}

In the previous section, the case of an ellipse was analyzed. Although the choice of this shape is ubiquitous in the experimental literature it is, from the theoretical point of view, a special case. This is because choosing the exponent to be exactly $\frac{1}{3}$ implies drawing with coupled harmonic motion. It is therefore worth examining another shape that has also been measured in experiments: the cloverleaf. This is a more interesting shape than an ellipse (no harmonic solution exists), but still shares the same property of a single segment on a $\log (\kappa)$ versus $\log (v)$ graph.

Unfortunately, the form of the cloverleaf used in experiment is far removed from a circle. Because of this, a similar perturbative approach to that used above yields bad results. For example, a general form for a cloverleaf that extrapolates between a circle $(\epsilon=0)$ and the figure used in experiment $(\epsilon=1)$ is:

$$
x=\cos 3 \theta(t)-\epsilon \cos \theta(t) \quad y=\sin 3 \theta(t)+\epsilon \sin \theta(t) .
$$
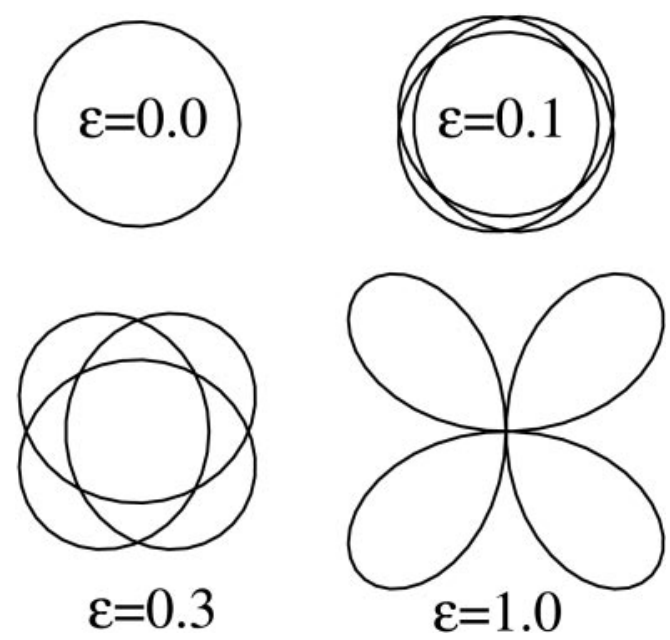

Figure 3. Examples of the generalized cloverleaf for various values of the perturbation parameter $\epsilon$. See Results (Eq. 11) for the mathematical definition.

Examples of this generalized cloverleaf for different values of $\epsilon$ are given in Figure 3. The perturbative expansion, used in the previous section and detailed in Appendix A, gives the following exponent for the cloverleaf:

$$
\beta=\frac{1530}{3631} \simeq 0.42
$$

which does not agree well with experiment: cloverleaves that were traced with periods of 2.5 and $3.0 \mathrm{sec}$ (Viviani and Flash, 1995) were found to have average values of $\beta$ of $0.35 \pm 0.03$ and $0.36 \pm$ 0.03 , respectively. (For completeness it should be noted that for faster tracing, with a period of $2 \mathrm{sec}$, the value was lower $\beta=$ $0.33 \pm 0.04$, but this was attributable to a somewhat-anomalous data point.) However, the inaccuracy of the result (Eq. 12) is a breakdown of the perturbative approach only. A numerical method can be used to obtain the velocity profile from the minimum-jerk cost function to an arbitrary degree of accuracy. Once the velocity profile has been obtained, the predicted exponent can be found from a least-squares fit of Equation 2: the same approach used for experimental velocity curves. Such a velocity curve was obtained from Equation 1, and the fit can be seen in Figure 4. The exponent obtained was:

$$
\beta=0.36 \pm 0.01
$$

which compares well with the two-thirds power law result and the experimental values given above. This result cannot be a trivial consequence of coupled harmonic motion, because for the cloverleaf there are no coupled harmonic solutions. The result therefore represents a success for the first-principles approach for calculating the exponent, particularly because the only input into the theory is the curvature profile given by Equations 11 .

\section{General one-segment shapes}

Two specific shapes were tested above. However, it is possible to generalize to an arbitrary one-segment shape. The approach is to write the curvature as a Taylor expansion in a small parameter $\epsilon$ and solve the optimization problem in terms of the derivatives of the curvature with respect to this variable. Details of the method 


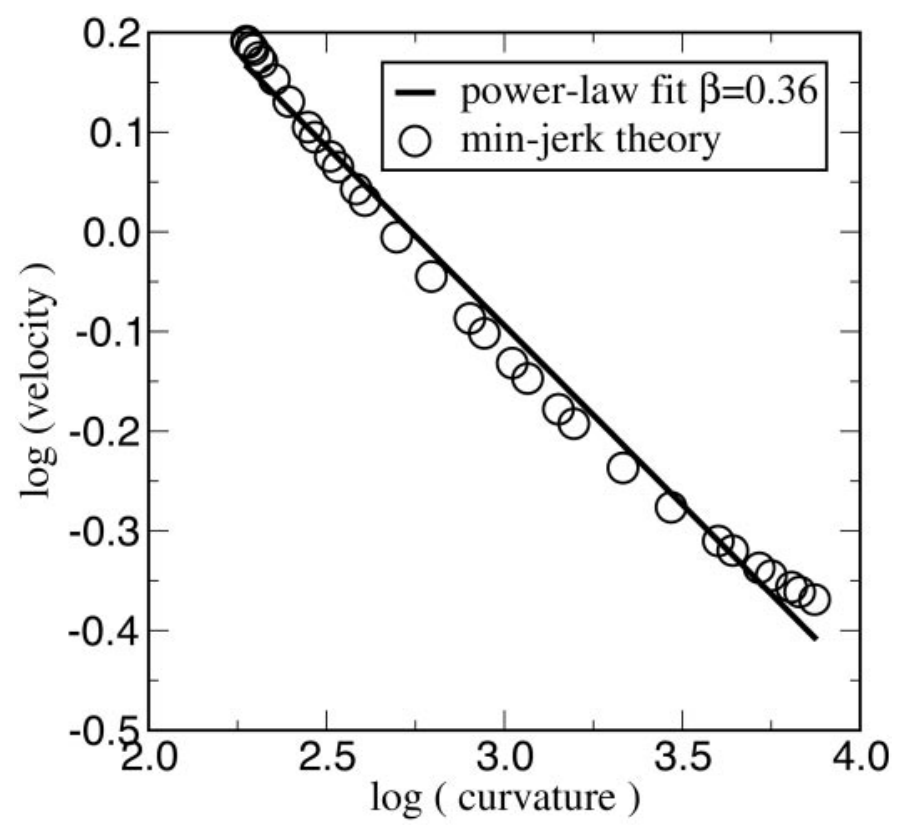

Figure 4. The $\log$ (curvature) versus $\log$ (velocity) curve as derived numerically from the minimum-jerk cost function for a cloverleaf with $\epsilon=$ 1 (in this case the units are arbitrary as the fit is to theory, not experiment). The value of the $\beta$ exponent measured from this curve is $\beta=0.36$, reproducing the results found in the experiment.

of obtaining this generalized form for the exponent $\beta$ are given in Appendix B.

\section{The drawing of complex figures}

In the previous section the cases of the ellipse and the cloverleaf were analyzed. Both of these shapes have a common feature: their curvature-velocity profiles are well matched by the twothirds power law with a single gain factor. This means that in a $\log -\log$ plot of the velocity versus curvature only a single segment is seen. In this section the more complex case of a multiplesegment shape is analyzed, specifically the asymmetric lemniscate, or figure-of-eight with two different sized lobes. Again comparison is made with experimental data, but it should be stressed that here quantitative accuracy is not required, just a qualitative reproduction of the features of segmentation. Because of the complexity of the analysis of multiple-segment shapes, the approach in this case is numerical. An algorithm similar to that described in Todorov and Jordan (1998) was used, in which the time the movement passes through 10 via-points is varied until the trajectory with the lowest minimum-jerk cost is found. The optimal times were obtained using a stochastic descent algorithm, to an accuracy of $0.25 \%$ of the total movement duration.

Before examining the results, it is worth underlining again the importance of using a first-principles approach in the derivation. In a related paper (Viviani and Flash, 1995), similar shapes were analyzed, but experimental information in the form of velocity and acceleration was also supplied at the via points. The argument put forward here is that the minimization of an integral cost function (e.g. minimum jerk) gives a movement that mimics the segmentation seen in experiment. This argument would be undermined if experimentally derived values of the velocity and curvature for the via-points were used as they could contain information about possible segmented planning of the movements by the CNS.

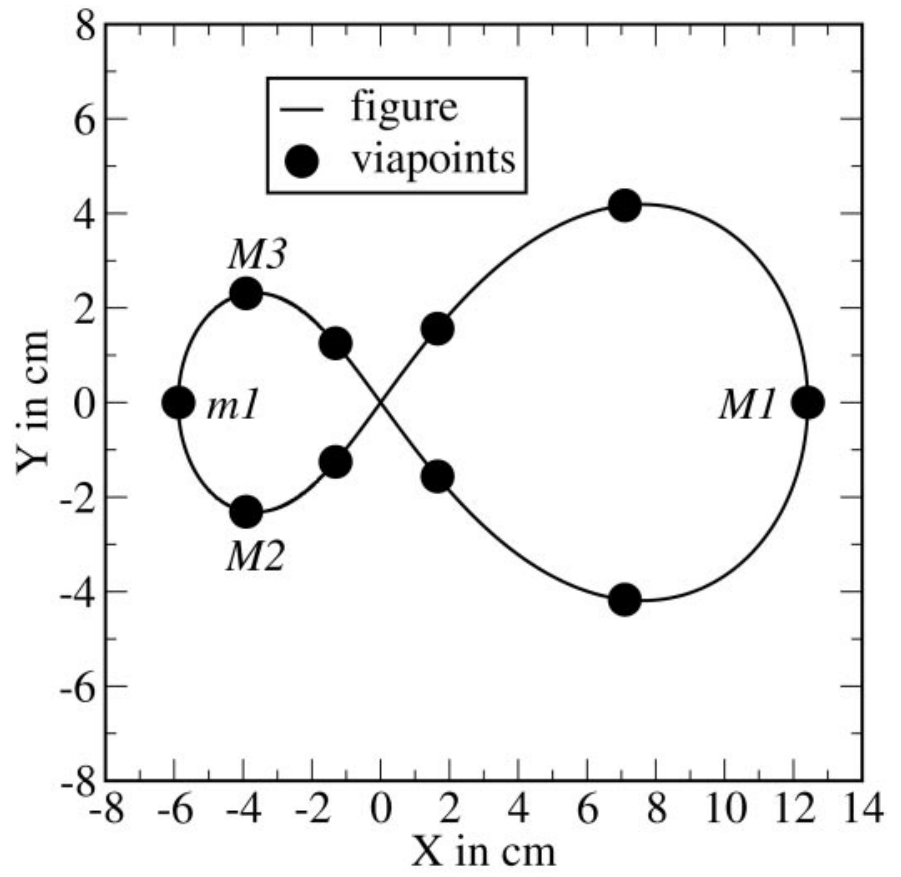

Figure 5. A plot of the figure-of-eight template. The numerical derivation of the minimum-jerk trajectory was obtained using 10 via-points. The labels $M 1, M 2$, and $M 3$ mark the curvature maxima, whereas $m 1$ is the curvature minimum. It is these extrema and their relation by symmetries that determine the number of apparent segments (Fig. 6).

\section{The figure-of-eight}

The shape to be analyzed has the following mathematical form:

$$
x=\frac{a(\cos \theta+b) \cos (\theta)}{1+\sin ^{2}(\theta)} \quad y=x \sin (\theta),
$$

with $a=3.26 \mathrm{~cm}$ and $b=2.80$ giving a large and a small lobe (Fig. $5)$. The drawing of shapes of this form has been extensively analyzed (Viviani and Flash, 1995), and it has been shown that when the lobes in the figure-of-eight have different sizes, the $\log$ (curvature) versus $\log$ (velocity) plots show two apparent segments (corresponding to the large and small lobes) with distinct gain factors. Using the form of the power law given by Equation 3 in Materials and Methods, measurements have shown (Viviani and Flash, 1995) that across a sample of three subjects the gain factors and power law fits were found to be:

$$
\begin{aligned}
& v=g_{L}\left(R^{*}\right)^{\beta_{L}} \text { with } g_{L}=21 \pm 3 \text { and } \beta_{L}=0.33 \pm 0.05 \\
& v=g_{S}\left(R^{*}\right)^{\beta_{S}} \text { with } g_{S}=17 \pm 3 \text { and } \beta_{S}=0.34 \pm 0.05
\end{aligned}
$$

for the large and small lobes, respectively (the gain factors are written in unitless form here), and the average period of tracing was $T=1.43 \mathrm{~s} \pm 0.05 \mathrm{~s}$. The errors in the gain factors mainly represent between-subject variance. Individually, the three subjects consistently traced the figure-of-eights with $g_{L}>g_{S}$ with ratios $g_{L} / g_{S}=\{1.35 \pm 0.04,1.23 \pm 0.03,1.12 \pm 0.03\}$. Thus, the experimental data can be interpreted as having two segments.

The numerically derived minimum-jerk trajectory is plotted in Figure 6 , in velocity $v$ versus $\left(R^{*}\right)^{1 / 3}$ form. In this format, different segments lie on radial lines passing through the origin, with gradients corresponding to the different gain factors $g_{L}$ and $g_{S}$. The duration of the movement has been scaled to agree with the average experimental period of $T=1.43 \mathrm{~s}$. As can be seen, the 


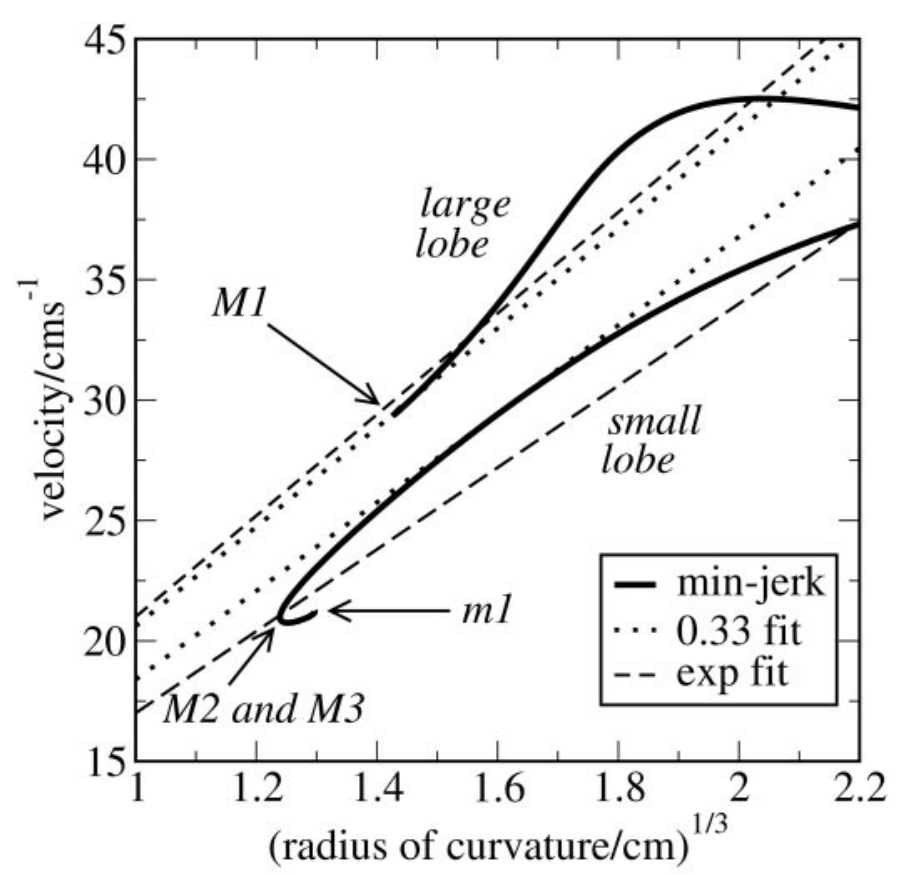

Figure 6. The predicted minimum-jerk velocity versus effective radius of curvature plot, for the tracing of the figure-of-eight. The positions of the curvature extrema $(M 1, M 2, M 3$, and $m 1)$ are marked and can be compared with Figure 5. The dotted tangential lines give an indication of the gain factors for the two segments (see Results), whereas the dashed lines represent the average experimental values. The theory reproduces the features of segmentation seen in the experimental curves, despite no explicit segmental planning in the structure of the theory.

theoretically derived curve shows qualitatively the same features of segmentation as the experimental data. The $\beta$ exponents of the power law are not too well defined for the theoretical curve because of the curvature of the two segments (unlike the experimental data, the theoretical curve has not been smoothed, a process which tends to increase the likelihood of measuring an exponent close to 0.33), and, as stressed above the aim here is to show that features of segmentation are seen in theoretical predictions, not to show close quantitative agreement. However, to provide a level of comparison, the tangential dotted lines in Figure 6 (meant as guides for the eye) represent gain factors corresponding to $g_{L}=20.5$ and $g_{S}=18.4$, which implies a ratio $g_{L} / g_{S}=1.1$ (the choice here of using tangents, rather than a fit, tends to underestimate the value of this ratio). Though this value is about $10 \%$ smaller than the average found experimentally $\left(\sim 1.2\right.$, the corresponding values of $g_{L}=21$ and $g_{S}=17$ are represented as dashed lines in Fig. 6), it is not inconsistent with the range of experimental values seen across the three subjects.

The theoretically generated trajectory captures all the qualitative features of segmentation, despite the absence of any piecewise algorithm in the minimum-jerk hypothesis. In fact even the hook-like feature (corresponding to the region of the trajectory between the curvature maxima $M 2$ and $M 3$ via the curvature minimum $m 1$ ) can be seen in a detailed analysis of the experimental data. In examining this feature, it becomes clear what criteria determine the apparent segmentation of the velocity versus curvature plots: it is the extrema of the curvature and the symmetries of the shape. Thus, the segmentation features seen in such log-log plots can be directly accounted for by the geometric aspects (curvature profile) of the drawn path: segmented planning in the CNS need not be assumed.

\section{DISCUSSION}

A family of movement-planning strategies for the human arm was analyzed in mathematical detail and compared with experiment, with a number of results derived. The planning strategies are defined through the minimization of cost functions that favor smoothness, to differing degrees, and are known collectively as the MSD cost functions. Two standard and contrasting motor tasks were analyzed: point-to-point reaching movements and the periodic tracing of closed shapes. For the first task of point-topoint movements the predicted velocity profile from each order $n$ of the MSD family was derived explicitly and compared with experiment. By comparing the ratio of the peak to average velocities it was concluded that the best fit (from the MSD family) was by the minimum-jerk cost function, corresponding to order $n=3$. It was further shown that as the order $n$ was increased the corresponding velocity profile diverged, reaching the highly unphysical limit of a Dirac delta function when $n \rightarrow \infty$. This result corrects conclusions previously reached in the literature (Harris, 1998). However, it is acknowledged here that this was not the main point of that paper, and therefore the current work should not be mistaken as a criticism of the minimum-variance principle, for which the paper of Harris (1998) laid the foundation. Rather, the aim here was to clarify the mathematical understanding of the smoothness-based cost functions.

The theory of minimum variance was applied to both arm and eye movements and may seem, at first view, to be at odds with the smoothness-based cost function approach. However, there are many overlaps between the two theories. An obvious example is the independence of the velocity profiles for fast movements from physical parameters of the arm. This suggests an interpretation of the minimum-jerk theoretical framework as a simplified limit of a more complete theory. As has already been noted, minimum-jerk predictions for point-to-point movements miss some of the fine structure of the trajectories. Experimentally measured trajectories show a small but systematic curvature, a feature captured by other models that take into account both the problems of trajectory planning and motor execution (Flash, 1987; Uno et al., 1989; Harris and Wolpert, 1998). In this context, it would be very interesting to examine theoretically the similarities between the MSD cost functions and other more detailed hypothesis such as the minimum-variance hypothesis (Harris and Wolpert, 1998) or the optimal feedback control hypothesis of Todorov (2001). In the latter work, it is demonstrated that greater control of a noisy goal-dependent variable can be obtained at the expense of allowing variance to increase in redundant degrees of freedom. In their formulation, features such as simplifying rules, control parameters, and synergies emerge as epiphenomena from the optimal feedback control.

Several neural implementations have been proposed for the minimum-jerk cost function (Hoff and Arbib, 1992; Jordan et al., 1994) and neurophysiological studies have shown that the fine kinematic details of the movements are represented within cortical neuronal populations (Schwartz and Moran, 1999). Nevertheless, given the present level of knowledge of the neural correlates of movement and also the success that different modeling approaches have in describing the same psychophysical experiments (as was shown here in the context of minimum-jerk and the two-thirds power law), it is clear that the data are not yet sufficiently fine to restrict the space of the possible neurally based algorithms that might generate the observed behavior. As an example, the optimal feedback hypothesis produces movements 
that are smooth, and in some limits may be reproduced by the minimum-jerk predictions, but the underlying structure of the controlling system is very different.

The second experimental task analyzed was the periodic drawing of closed shapes. In contrast to point-to-point movements the order- $n$ velocity profile was shown to converge to a smooth function as $n$ was increased. For the limit $n \rightarrow \infty$ it was shown that the predicted velocity profile is the one for which the Fourier series for the trajectory of the hand has the lowest values in the high-frequency range. For the specific examples of an ellipse and cloverleaf it was shown that the power-law exponent derived from a MSD cost function, with any order greater than minimum acceleration, gives values that are indistinguishable from the accepted value of $\beta \simeq 0.33$. Further comparison was then made between the two theories for the case of a complex figural form (a figure-of-eight with different sized lobes). The velocity profile derived from the MSD smoothness-based cost functions was seen to have features that implied segmented control. However, the cost function contains no explicit segmented control. Therefore, the conclusion must be that these types of experimental features can no longer be taken as evidence for segmented control in the $\mathrm{CNS}$, in the absence of further supporting evidence.

Despite the success of the smoothness-based cost function in fitting different aspects of the two-thirds power law, there are still many questions unanswered. In earlier studies several different reasons and explanations for the observed two-thirds power law were offered. These reasons included either the mechanical properties of muscles (Gribble and Ostry, 1996), acceleration, or noise constraints (Harris and Wolpert, 1998) or the coupling between oscillatory joint rotations (Sternad and Schaal, 1999). The analysis performed by Schwartz (1994) and more recently by Schwartz and Moran (1999), has shown that the two-thirds power law fits neural trajectories derived by using a population vector analysis of the neural activity data recorded from motor cortical cells in monkeys during drawing movements. The fact that the two-thirds power law has been found in motor cortical representations of drawing movements suggests that this relation is manifested in the planning stages of hand trajectories (Schwartz and Moran, 2000), consistent with the idea that central neural representations have evolved or are acquired with age (Viviani and Schneider, 1991) or through learning to achieve the smoothest and/or the least variable movement. Nevertheless, explanations for the twothirds power law based on central mechanisms or on peripheral factors, such as the mechanical properties of muscles or neuromotor noise, may not be mutually exclusive, and both peripheral and central factors might have evolved to work in tandem to guarantee the smoothest possible or most accurate movement. Other questions of interest that relate to the work presented here concern the nature of the relation between the two-thirds power law and visual perception of motion. There is compelling evidence from a number of studies that the two-thirds power law is related to both the motor production and visual perception of movement (Pollick and Sapiro, 1996; de'Sperati and Viviani, 1997). Such connections were not examined here and represent an interesting case for further study.

Another significant topic for future study that was addressed here is that of movement segmentation. Many earlier hypotheses concerning the nature of the underlying movement segments have been made (Krebs et al., 1999; Doeringer and Hogan, 1998). Among them, one of the most compelling hypotheses, which attempted to suggest a criterion for motion segmentation, is that based on the existence of a piecewise constant gain factor in the relation between velocity and curvature (Lacqaniti et al., 1983). Using a similar approach, the boundaries of movement segments during drawing movements were defined kinematically as places in the trajectory where speed is maximal (Schwartz and Moran, 1999). These are points at which the velocity gain factor in the power law changes instantaneously, where curvature is minimal, and where curvature inflections also occur. The conclusion, based on the mathematical analysis presented here is that the presence of sharp changes in the velocity gain factor does not necessarily suggest a noncontinuous mode of motion planning and therefore cannot provide sufficient evidence for segmented control [supporting the findings reached in the experimental study of Sternad and Schall (1999)]. This conclusion does not undermine the idea that complex movements are constructed from simpler units of action or movement segments that are then concatenated together or are temporally overlapped to generate longer movement sequences. It does, however, raise the need for new ideas concerning the nature of the underlying movement segments or strokes.

\section{APPENDIX A}

This appendix contains the details of the calculation leading to the predicted exponent for the power law of the ellipse, Equation 10 , as derived from the general order- $n$ MSD cost function. One route to obtaining the exponent $\beta_{n}$ of the power law is to substitute the velocity form given in Equation 2 into the integral (Eq. 1) and change the integration variable from time to arc-length. This can be done directly for very small values of $n$ by using the Frenet-Serret formulation for curves (see Appendix B). However, to get general results for arbitrary order, a less direct approach is necessary. The route taken to find $\beta_{n}$ is to obtain the Fourier coefficients for the cost function (Eq. 7) in terms of the unknown exponent $\beta$. Once this has been done it is straightforward to minimize $C_{n}$ by differentiation with respect to the exponent.

The first step is to write the coordinates of the hand (quite generally) as parametric functions of a time dependent angle $\theta(\tau)$ :

$$
x(\tau)=A \cos \theta(\tau) \quad y(\tau)=B \sin \theta(\tau),
$$

with boundary conditions $\theta(0)=0, \theta(1)=2 \pi$ and $\tau=t / T$, where $T$ is the period. Next, the time dependence of the angle must be found by using the power law (Eq. 2). It is the time dependence of $\theta$ that encodes the velocity profiles. Once this is done the Fourier coefficients required for the cost function can be easily derived. Writing the velocity as the rate-of-change of arc-length $s$ with respect to time allows Equation 2 to be rearranged to give:

$$
T d \tau=\frac{d s}{g} \kappa^{\beta}=\frac{d \theta}{g}\left(\frac{d s}{d \theta}\right) \kappa^{\beta} .
$$

The integral can now be performed, and the gain factor $g$ is fixed by the condition that $\theta=2 \pi$ when normalized time $\tau=1$ :

$$
\tau=\frac{\int_{0}^{\theta} d \theta\left(\frac{d s}{d \theta}\right) \kappa^{\beta}}{\int_{0}^{2 \pi} d \theta\left(\frac{d s}{d \theta}\right) \kappa^{\beta}}=\frac{\int_{0}^{\theta} d \theta(1-\epsilon \cos \theta)^{\frac{1-3 \beta}{2}}}{\int_{0}^{2 \pi} d \theta(1-\epsilon \cos \theta)^{\frac{1-3 \beta}{2}}} .
$$


In the second equation, the following geometric properties of the ellipse have been used:

$$
\kappa=A B\left(\frac{d s}{d \theta}\right)^{-3} \quad\left(\frac{d s}{d \theta}\right)=A\left(1-\epsilon \cos ^{2} \theta\right)^{1 / 2} \quad \epsilon=1-B^{2} / A^{2} .
$$

The quantity $\epsilon$ is related to the eccentricity of the ellipse and is a measure of how close the ellipse in question is to a circle, for which $\epsilon=0$. To obtain the time $\tau$ as a function of angle, the integrals in Equation 15 must be performed. Unfortunately, this is not possible exactly because of the nature of the integrals and to proceed further it is necessary to make an approximation. It will be assumed now that the quantity $\epsilon$ is very small (more will be said about the validity of this approximation later). This allows the arguments of the integrals to be expanded as a series in the small parameter $\epsilon$ using standard formulas, and then the integration performed. The result is also a series expansion in $\epsilon$ which, in all the following steps, will be written up to order $\epsilon^{2}$ :

$$
\begin{aligned}
\tau=\frac{1}{2 \pi}\left(\theta+\frac{\epsilon}{8}(3 \beta-1)\right. & \sin 2 \theta \\
& \left.+\frac{\epsilon^{2}}{256}\left(16 \sin 2 \theta+\left(9 \beta^{2}-1\right) \sin 4 \theta\right)\right)+\epsilon^{3} \cdots
\end{aligned}
$$

This expansion can now be inverted to give the angle as a function of time, the quantity needed for Equations 14. The inversion can be performed by substituting the following into the previous equation:

$$
\theta(\tau)=f(\tau)+\epsilon g(\tau)+\epsilon^{2} h(\tau)+\epsilon^{3} \cdots
$$

The trigonometric functions can then be expanded again, keeping terms only up to order $\epsilon^{2}$. Then by matching terms with the same powers of $\epsilon$ the following functions of time are found:

$f(\tau)=2 \pi \tau$

$g(\tau)=\frac{1}{8}(1-3 \beta) \sin 4 \pi \tau$

$h(\tau)=\frac{1}{16}(1-3 \beta) \sin 4 \pi \tau+\frac{1}{256}(3 \beta-1)(9 \beta-5) \sin 8 \pi \tau$.

As expected, the first contribution $f(\tau)$ is the result for the angular dependence of time when a circle is drawn. The higher order terms $g(\tau)$ and $h(\tau)$ represent the corrections caused by the elliptical perturbation.

What remains now is to obtain the Fourier coefficients in Equation 6 of the $x$ and $y$ coordinates. This is achieved by substituting Equation 16 with the results (Eq. 17) into Equation 14, for example:

$$
a_{k}=2 \int_{0}^{1} d \tau A \cos \left(f(\tau)+\epsilon g(\tau)+\epsilon^{2} h(\tau)\right) \cos 2 \pi k \tau+\epsilon^{3} \ldots
$$

The argument of the cosine can be expanded in $\epsilon$ allowing the integral to be performed and the Fourier coefficients obtained, accurate to order $\epsilon^{2}$. The calculation is similar for $b_{k}, p_{k}$, and $q_{k}$, although for the latter two it must be remembered that $B=A(1-$ $\epsilon)^{1 / 2}$ and that this should also be expanded to the appropriate order. The final results for the squares of the Fourier coefficients are:

$$
\begin{aligned}
& a_{1}^{2}=A^{2}\left(1+\frac{\epsilon}{16}(3 \beta-1)+\frac{\epsilon^{2}}{256}\left(30 \beta-9 \beta^{2}-9\right)\right)^{2}+\epsilon^{3} \cdots \\
& a_{3}^{2}=A^{2} \frac{\epsilon^{2}}{256}(1-3 \beta)^{2}+\epsilon^{3} \cdots \\
& q_{1}^{2}=A^{2}\left(1+\frac{\epsilon}{16}(3 \beta-1)+\frac{\epsilon^{2}}{256}\left(30 \beta-9 \beta^{2}-9\right)\right)^{2}+\epsilon^{3} \ldots \\
& q_{3}^{2}=A^{2} \frac{\epsilon^{2}}{256}(1-3 \beta)^{2}+\epsilon^{3} \cdots
\end{aligned}
$$

where all other terms are either zero or small enough to be neglected (order $\epsilon^{3}$ or higher). At this level, only the first two terms in the infinite series for the cost function (Eq. 7) remain:

$$
C_{n}=\frac{T}{2}\left(\frac{2 \pi}{T}\right)^{2 n}\left[\left(a_{1}^{2}+q_{1}^{2}\right)+9^{n}\left(a_{3}^{2}+q_{3}^{2}\right)\right]+\epsilon^{3} \cdots .
$$

Substituting in the Fourier coefficients and differentiating with respect to $\beta$, while equating the resulting expression to zero, yields an equation for the predicted power-law exponent from the order- $n$ MSD hypothesis, which is accurate at a level of $\epsilon^{2}$ :

$$
\frac{d C_{n}}{d \beta}=\frac{\epsilon^{2} A^{2} T}{128}\left(\frac{2 \pi}{T}\right)^{2 n}\left(27-9 \beta_{n}+9^{n}\left(3 \beta_{n}-1\right)\right)=0 .
$$

The exponent $\beta_{n}$ should of course also be written as an expansion in $\epsilon$. However, there is a prefactor of $\epsilon^{2}$ in the equation above. So even though the calculation has been performed throughout at the level of $\epsilon^{2}$, it is only possible to obtain the $\epsilon^{0}$ order term of the $\beta_{n}$ expansion. The result is:

$$
\beta_{n}=\frac{1}{3}-\frac{8}{3\left(9^{n}-1\right)}+\text { corrections of order } \epsilon^{2} .
$$

Because the approach used involved an approximation (ellipses that were similar to circles were considered), the validity of the perturbation expansion must be examined. As stated above, the exponent derived is just the first term in an $\epsilon$ expansion. The result is therefore only strictly valid for ellipses that have low eccentricity. However, the size of the first correction to $\beta_{n}$ can be calculated by repeating the whole of the last derivation but this time keeping terms up to order $\epsilon^{4}$. As an example for minimum jerk, the first correction is only $0.000336 \epsilon^{2}$ : there is no term at the level of $\epsilon$, and the correction at $\epsilon^{2}$ is clearly small. Therefore, despite the approximation, the results derived are valid for ellipse eccentricities of similar magnitude to those that were used in experiment, because of this small correction term.

\section{APPENDIX B}

The first step is to re-write the cost function (Eq. 1) with $n=3$ for minimum jerk in terms of velocity and curvature. This step can be done conveniently by using Frenet's relations, see for example Todorov and Jordan (1998). The result is:

$$
C=\int_{0}^{L} \frac{d s}{v}\left(\left(v^{2} v^{\prime \prime}+v\left(v^{\prime}\right)^{2}-\kappa^{2} v^{3}\right)^{2}+\left(3 v^{\prime} v^{2} \kappa+v^{3} \kappa^{\prime}\right)^{2}\right)
$$

where $s$ is the arc-length, $L$ is the circumference of the path, $\kappa$ is the curvature, and in this equation the notation $X^{\prime}$ means $X$ 
differentiated with respect to arc-length. As in Appendix A, the gain factor $g$ must be fixed by the restriction that all trajectories have the same period:

$$
\int_{0}^{L} \frac{d s}{v}=T
$$

This gives the following form for the gain factor (using $v=g \kappa^{-\beta}$ ):

$$
g=\int_{0}^{2 \pi} d \theta \xi \kappa^{\beta} \text { where } \xi=\frac{d s}{d \theta}
$$

where from now on the scaling choice has been made that $T=$ $L=1$. This is merely a choice of units of time and length and does not affect the generality of any of the following results. It should be noted that there are two dependencies on $\beta$ : in the gain factor $g$ and also in the form $\kappa^{-\beta}$ :

$$
v=\left(\int_{0}^{2 \pi} d \theta \xi \kappa^{\beta}\right) \kappa^{-\beta}
$$

which complicates the calculation. When the cost function is written in terms of an integration over angle, by using $d s=\xi d \theta$, it has the form:

$$
C=\int_{0}^{2 \pi} d \theta F(v, \kappa, \xi)
$$

where all the dependence on the exponent $\beta$ is contained in the velocity $v$. In general it is still very difficult (if not impossible) to minimize this cost function with respect to $\beta$ exactly. It is therefore necessary to proceed with an approximation.

Again, perturbations from a circle to a shape of interest are considered. By symmetry the extremizing velocity is a constant for a circle, and hence this particular example is easy to solve. It is assumed that there exists some parameter $\epsilon$ that measures how close the shape in question is to a circle, for which $\epsilon=0$. The curvature and differential $\xi$ can be expanded in terms of this parameter:

$$
\kappa=\kappa_{0}\left(1+\epsilon \kappa_{1}+\cdots\right) \quad \xi=\xi_{0}\left(1+\epsilon \xi_{1}+\cdots\right),
$$

where $\kappa_{0}$ and $\xi_{0}$ are the values for a circle and the higher order expansion terms (like $\kappa_{1}$ and $\xi_{1}$ ) are angle dependent and specific to the shape in question. The method to be followed now is to substitute these expansions into the cost function and expand in a power series $C=C_{0}+C_{1} \epsilon+C_{2} \epsilon^{2} \cdots$ (where here the subscripts refer to the order of the expansion: all the calculations here are for minimum jerk only):

$$
C_{1}=\left.\frac{d C}{d \epsilon}\right|_{0}=\left.\frac{\partial F}{\partial v}\right|_{0} \int_{0}^{2 \pi} \frac{d v}{d \epsilon}\left|{ }_{0} d \theta+\frac{\partial F}{\partial \kappa}\right|_{0} \int_{0}^{2 \pi} \kappa_{1} d \theta+\left.\frac{\partial F}{\partial \xi}\right|_{0} \int_{0}^{2 \pi} \xi_{1} d \theta,
$$

with the notation $\left.X\right|_{0}$ representing the quantity $X$ evaluated for the circular case, i.e. with $\epsilon=0$. To obtain the predicted exponent, this expansion must be minimized:

$$
\frac{d C}{d \beta}=\frac{d C_{0}}{d \beta}+\epsilon \frac{d C_{1}}{d \beta}+\epsilon^{2} \frac{d C_{2}}{d \beta} \cdot \cdots=0 .
$$

Minimizing with respect to $\beta$ at some order of perturbation theory will then give the exponent at that level of approximation. Clearly the first term of Equation 20 is zero because this is simply the cost for a circle and must be independent of $\beta$. It can be shown that $C_{1}$ is also independent of $\beta$, and in fact the first contribution to Equation 20 appears only at order $\epsilon^{2}$. Therefore, the following must be solved:

$$
\epsilon^{2} \frac{d}{d \beta} C_{2}\left(\left\{\kappa_{0}, \xi_{0}, \kappa_{1}, \cdots\right\}, \beta\right)=0,
$$

which requires a full evaluation of the integral $C$ keeping all terms at order $\epsilon^{2}$ throughout. It should also be noted that because of the prefactor $\epsilon^{2}$ in the equation above, the solution for the exponent will only be the zero-order term in an $\epsilon$ expansion for $\beta$.

Because no specific values of the curvature or differential $\xi$ have been specified, the method described here is quite general. After some algebra, the minimizing $\beta$ can be found and has the following value:

$$
\beta=\frac{10 \overline{\Delta^{2}}+5 \eta^{2} \overline{\dot{\Delta}^{2}}}{15 \overline{\Delta^{2}}+15 \eta^{2} \overline{\dot{\Delta}^{2}}+\eta^{4} \overline{\Delta^{2}}},
$$

where the notation used is defined as follows:

$$
\begin{array}{lr}
\eta=\left(\kappa_{0} \xi_{0}\right)^{-1} & \Delta=\kappa_{1}-\bar{\kappa}_{1} \\
\dot{X}=\frac{d X}{d \theta} & \bar{X}=\frac{1}{2 \pi} \int_{0}^{2 \pi} d \theta X(\theta) .
\end{array}
$$

It should be noted that the exponent is a function of only $\left\{\kappa_{0}, \kappa_{1}\right.$, $\left.\xi_{0}\right\}$. The quantity $\eta$ is unity for shapes that are deformation of a circle drawn once (like the ellipse). However, it takes different values for more complex cases like the cloverleaf. The result derived above can be used for any one-segmented shape (in the power-law sense) that is sufficiently close to a circle.

\section{REFERENCES}

de'Sperati C, Viviani P (1997) The relationship between curvature and velocity in two-dimensional smooth pursuit eye movements. J Neurosci 17:3932-3945.

Doeringer JA, Hogan N (1998) Serial processing in human movement production. Neural Networks 11:1345-1356.

Flash T (1987) The control of hand equilibrium trajectories in multijoint arm movements. Biol Cybern 57:257-274.

Flash T, Hogan N (1985) The coordination of arm movements: an experimentally confirmed mathematical model. J Neurosci 5:1688-1703.

Gribble PL, Ostry DJ (1996) Origin of the power law relation between movement velocity and curvature: modelling the effects of muscle mechanics and limb dynamics. J Neurophysiol 76:2853-2860.

Harris CM (1998) On the optimal control of behavior: a stochastic perspective. J Neurosci Methods 83:73-88.

Harris CM, Wolpert DM (1998) Signal-dependent noise determines motor planning. Nature 394:780-784.

Hoff B, Arbib MA (1992) A model of the effects of speed, accuracy, and perturbation on visually guided reaching. In: Control of arm movement in space: neurophysiological and computational approaches (Caminiti $\mathrm{R}$, Johnson PB, and Burnod Y, eds), pp 285-306.

Hogan N (1984) An organizing principle for a class of voluntary movements. J Neurosci 4:2745-2754

Jordan MI, Flash T, Arnon Y (1994) A model of the learning of arm trajectories from spatial deviations. J Cognit Neurosci 6:359-376.

Krebs HI, Aisen ML, Volpe BT, Hogan N (1999) Quantization of continuous arm movements in humans with brain injury. Proc Natl Acad Sci USA 96:4645-4649.

Lacquaniti F, Terzuolo C, Viviani P (1983) The law relating the kinematics and figural aspects of drawing movements. Acta Psychol 54:115-130.

Morasso P (1981) Spatial control of arm movements. Exp Brain Res 42:223-227.

Nakano E, Imamizu H, Osu R, Uno Y, Gomi H, Yoshioka T, Kawato M (1999) Quantitative examinations of internal representations for arm 
trajectory planning: minimum commanded torque change model. J Neurophysiol 81:2140-2155.

Plamondon R, Alimi AM, Yergeau P, Leclerc F (1993) Modeling velocity profiles of rapid movements: a comparative study. Biol Cybern 69:119-128.

Pollick FE, Sapiro, G (1997) Constant affince velocity predicts the $1 / 3$ power law of planar motion perception and generation. Vision Res 37:347-353.

Schwartz AB (1994) Direct cortical representations of drawing. Science 265:540-542.

Schwartz AB, Moran DW (1999) Motor cortical activity during drawing movements: population representation during leminiscate tracing. J Neurophysiol 82:2705-2718.

Schwartz AB, Moran DW (2000) Arm trajectory and representation of movement processing in motor cortical activity. Eur J Neurosci 12:1851-1856.

Sternad D, Schaal S (1999) Segmentation of endpoint trajectories does not imply segmented control. Exp Brain Res 124:118-136.

Todorov E (2001) Optimal feedback control as a theory of motor coordination. Soc Neurosci Abstr 27:301.8.

Todorov E, Jordan MI (1998) Smoothness maximization along a pre- defined path accurately predicts the speed profiles of complex arm movements. J Neurophysiol 80:696-714.

Uno Y, Kawato M, Suzuki R (1989) Formation and control of optimal trajectories in human multijoint arm movements: minimum torquechange model. Biol Cybern 61:89-101.

Viviani P, Cenzato M (1985) Segmentation and coupling in complex movements. J Exp Psychol 6:828-845.

Viviani P, Flash T (1995) Minimum-jerk, two-thirds power law, and isochrony: converging approaches to movement planning. J Exp Psychol 21:32-53.

Viviani P, Schneider R (1991) A developmental study of the relationship between geometry and kinematics in drawing movements. J Exp Psychol 17:198-218.

Viviani P, Stucchi N (1992) Biological movements look constant: evidence of motor perceptual interactions. J Exp Psychol 18:603-623.

Viviani P, Terzuolo C (1982) Trajectory determines movement dynamics. Neuroscience 7:431-437.

Wann J, Nimmo-Smith I, Wing AM (1988) Relation between velocity and curvature in movement: equivalence and divergence between a power law and a minimum-jerk model. J Exp Psychol 14:622-637. 